\title{
Lavori ancora in corso. Spunti di riflessione tra Porta e Manzoni
}

\author{
Federica Alziati \\ Université de Fribourg / Università Cattolica del Sacro Cuore di Milano
}

\begin{abstract}
A più di un decennio dalla pubblicazione della silloge di Dante Isella, Carlo Porta: cinquant'anni di lavori corso, il cantiere degli studi portiani deve considerarsi tutt'altro che concluso. E uno dei campi d'indagine ancora aperti è senz'altro l'ambito dei legami tra l'universo di Porta e la rivoluzione romantica e realista operata dal romanzo di Manzoni. Alla messa a fuoco della realtà umana che popola le pagine di entrambi si proverà minimamente a contribuire con una serie di accostamenti (alcuni noti, molti altri di fatto inediti) che ne facciano risaltare l'intima solidarietà.
\end{abstract}

Keywords: Alessandro Manzoni, Carlo Porta, I promessi sposi, romanticismo milanese, letteratura dialettale

Agli esordi del nuovo millennio Dante Isella suggellava il proprio impegno di editore e studioso tanto di Porta che di Manzoni inaugurando, da un lato, la maggiore impresa editoriale manzoniana dei nostri giorni ${ }^{1}$ e radunando, dall'altro, alcuni dei suoi principali contributi portiani sotto un titolo significativamente dispiegato a consegnare l'eredità del passato all'incalzare del futuro: Carlo Porta: cinquant'anni di lavori in corso. ${ }^{2} \mathrm{E}$ se è indubbio che le edizioni critiche del Fermo e Lucia e degli Sposi promessi abbiano infuso un rinnovato vigore agli sforzi dei manzonisti, sarà per lo meno lecito immaginare tutt'oggi aperto anche il cantiere portiano e auspicare che si moltiplichino in esso gli spiragli di attività. ${ }^{3}$ In una simile prospettiva,

I Si intende, ovviamente, il progetto filologico sfociato sinora nella pubblicazione di Alessandro Manzoni, Fermo e Lucia. Prima minuta (I821-I823), testo e apparato critico a cura di Barbara Colli, Paola Italia e Giulia Raboni, Milano, Casa del Manzoni, 20o6; e di Alessandro Manzoni, Gli sposi promessi (I823-I827), testo e apparato critico a cura di Barbara Colli e Giulia Raboni, Milano, Casa del Manzoni, 2012. A queste edizioni si farà riferimento per le citazioni dal Fermo e dalla seconda minuta.

2 Dante Isella, Carlo Porta: cinquant'anni di lavori in corso, Torino, Einaudi, 2003. Tra gli altri interventi si segnala almeno la raccolta di saggi I Lombardi in rivolta. Da Carlo Maria Maggi a Carlo Emilio Gadda, Torino, Einaudi, 1984 (in parte confluita nell'ultima silloge); sul fronte dei lavori editoriali di ambito portiano s'impongono invece l'edizione critica delle Poesie, Firenze, La Nuova Italia, 1956; l'edizione delle Lettere di Carlo Porta e degli amici della Cameretta, Milano-Napoli, Ricciardi, I967; e la curatela del volume delle Poesie, Milano, I Meridiani Mondadori, I975 [poi 2000].

3 Nella bibliografia più recente su Carlo Porta si registrano in particolare le edizioni commentate dei Poemetti, a cura di Guido Bezzola, Venezia, Marsilio, 1997; e delle Poesie, a cura di Pietro Gibellini, traduzione e note di Massimo Migliorati, Milano, Mondadori, 20II; così come 
un campo fra i più battuti ma ancora in parte da dissodare risulta, inevitabilmente, proprio la dinamica dei rapporti con la figura che agli occhi dei posteri incarna il centro gravitazionale della stagione romantica milanese: mentre il nome di Manzoni esercita, com'è logico, una comprensibile forza di attrazione nell'ambito dei commenti al Porta, quest'ultimo riaffiora invece troppo di rado nella bibliografia manzoniana, considerando la successione cronologica dell'esperienza dei due autori, delle rispettive opere e delle comuni amicizie intellettuali e letterarie. ${ }^{4}$ E non si tratterà, per gli studiosi, di provare ad esaurire il catalogo dei debiti espliciti o delle riprese dirette da parte di Manzoni, ma di muovere dagli accostamenti linguistici e stilistici e dalle sintonie di contenuto per esplorare il più vasto orizzonte di un'appartenenza sociale, culturale e artistica innegabilmente condivisa. ${ }^{5}$ Anche le nuove spigolature che si intendono proporre qui di seguito guadagneranno allora significato man mano che si svincoleranno da un'impostazione compilatoria per andare a ricomporsi nella fisionomia sincera, al contempo comica e dolente, dell'umanità portata alla ribalta con coraggio sia dal poeta che dal romanziere, dai crocicchi milanesi al confluire di stradicciole di campagna, dal dialetto meneghino a una lingua finalmente rivolta al più vasto pubblico nazionale, quasi in un inevitabile e fecondo passaggio di consegne. ${ }^{6}$

la monografia di Mauro Novelli, Divora il tuo cuore, Milano. Carlo Porta e l'eredità ambrosiana, Milano, Il Saggiatore, 2013.

4 Nell'impossibilità di mettere a tema in questa sede caratteri e protagonisti del romanticismo milanese, di affrontare il problema dei legami tra Porta e Manzoni o delle intersezioni tra la Cameretta portiana e lo studiolo di via del Morone (entrambi frequentati, com'è noto, da interlocutori privilegiati dell'uno e dell'altro autore, come Tommaso Grossi, Gaetano Cattaneo o Ermes Visconti), si rimanda ai contributi in materia che derivano dalle introduzioni e dagli apparati di commento di Gibellini, Bezzola e Isella alle edizioni portiane sopra citate; non si può inoltre prescindere dal fondamentale saggio di Dante Isella, Porta e Manzoni, Porta in Manzoni, in Id., Carlo Porta..., cit., pp. 238-284; si devono infine annoverare anche studi più datati come il volume di Guido Bezzola, Vita di Carlo Porta nella Milano del suo tempo, Milano, Rizzoli, I980; la raccolta La poesia di Carlo Porta e la tradizione milanese (Atti del Convegno di studi organizzato dalla Regione Lombardia, Milano I6-I8 ottobre 1975), Milano, Feltrinelli, 1976, in particolare il saggio di Ettore Bonora, Prestiti portiani nei "Promessi Sposi", alle pp. 195202; dello stesso autore, Osservazioni sui lombardismi dei "Promessi Sposi", in Ettore Bonora, Manzoni. Conclusioni e proposte, Torino, Einaudi, I976, pp. I25-I6I; o il breve ma interessante articolo di Giorgio Bassani, Manzoni e Porta, in «Paragone», 78, 1956, pp. 36-39.

5 Ben sintetizza Isella, al riguardo, indicando che «la corrispondenza tra la pagina manzoniana e le poesie del Porta sono come le somiglianze di chi fa parte della stessa famiglia» e che «di fronte ai testi del Porta il Manzoni non opera [...] per semplice ricalco, me se ne serve come di un modello generativo» (Isella, Porta e Manzoni..., cit., pp. 250 e 26I-262).

6 Difficile sottrarsi all'eco di un suggestivo appunto di Angelo Stella: «Nessuno, come il romantico Manzoni, mostra di raccogliere le ragioni profonde della poesia portiana, che aveva attraversato l'esperienza giacobina e napoleonica per ricuperare un referente popolare a una letteratura finita nell'Accademia. Ma perché i desgrazzi dei vari Bongee non si ripropongano all'infinito, era necessario - secondo il Manzoni - che costoro si impadronissero della lingua: 
Ad affacciarsi per primo ecco dunque Giovannin Bongee, che col racconto accorato delle sue ripetute Desgrazzi ha ispirato a diversi interpreti un parallelo con il don Abbondio manzoniano e le disavventure che inaugurano I promessi sposi. ${ }^{7} \mathrm{E}$ davvero il suo ingresso in scena ( $\mathrm{E}$ andava inscì bell bell come se fa / Ziffoland de per mì sulla mia dritta») richiama le movenze del curato che "tornava bel bello dalla passeggiata verso casa», ${ }^{8}$ mentre il lamento che scandisce le sue seconde peripezie pare anticipare il sistema di vita di quest'ultimo:

[...] quand gh'è di rogn tra quajghedun,

Regola ginerala ficchi el vell,

Chè no vuj minga per on gust de ciall

Andà in peltrera o toeù su el reff in fall.

Eppur con tutt el mè fa de cojon,

Col mè bon pè de piombi e l'arà drizz,

Giust perchè son nassuu in quel dì birbon

Ogni bott borli dent in quaj pastizz. ${ }^{9}$

Accettando di seguire ancora per un tratto la passione oratoria di Giovannin e il suo inascoltato desiderio di giustizia ci si troverà, tuttavia, ben presto indirizzati anche sulle orme di Renzo e delle sue peripezie milanesi. ${ }^{10}$

che diventassero Lorenzo Tramaglino» (Angelo Stella, Un "filo lombardo". Da Bonvesin, al Porta, al Manzoni, in La poesia di Carlo Porta e la tradizione milanese..., cit., p. 46).

7 Così già Attilio Momigliano, L'opera di Carlo Porta. Studio compiuto sui versi editi e inediti, Città di Castello, Lapi, 1909, pp. 42-58; ma anche Bassani, Manzoni e Porta, cit., p. 38; e infine Isella, Porta e Manzoni..., cit., pp. 253-254.

8 Desgrazzi de Giovannin Bongee (I8I2), vv. 8-9 («e andavo così bel bello come si fa, zufolando tra me e me sulla mia destra»: l'edizione di riferimento per la lezione, la traduzione e la cronologia testi portiani è il meridiano a cura di Isella sopra citato) e Promesssi Sposi I 8 (citati da Alessandro Manzoni, I promessi sposi e Storia della colonna infame, edizione critica dell'edizione definitiva I840-I842 a cura di Luca Badini Confalonieri, Roma, Salerno Editrice, 2006); i due passi sono stati accostati in particolare nei commenti al romanzo di Biancamaria Travi (Milano, Mondadori, I98I, ad locum) e di Salvatore Silvano Nigro (Milano, I Meridiani Mondadori, 2002, ad locum).

9 Olter desgrazzi de Giovannin Bongee (I8I3-18I4), vv. 2I-28 («quando ci sono delle questioni tra qualcuno io, per regola generale, prendo il largo, perché non voglio mica per un gusto da scemo finire sul banco degli imputati o prendermi per sbaglio un gran spavento. | Eppure, con tutto il mio far finta di niente e il mio buon piede di piombo e l'arare dritto, giusto perché sono nato in quel giorno birbone, ogni tanto ruzzolo dentro qualche pasticcio»); i vv. 2I-22 sono paragonati alle regole del curato nel commento ai Promessi sposi a cura di Ezio Raimondi e Luciano Bottoni (Milano, Principato, 1988, ad locum). L'incipit del testo portiano ("Quand se nass deslippaa, Lustrissem scior», v. I) potrebbe invece essere tradotto con l'esclamazione che sfugge al notaio in PS XV 43: «Quando uno nasce disgraziato!».

Io Un suggerimento in tal senso potrebbe venire da alcune considerazioni di Gibellini: «la fisionomia di Giovannin è ancora velata dalla maschera caricaturale ereditata dalla tradizione del popolano millantantore e bastonato, da satira del villano trasferita dal contado alla città. 
Al pari del montanaro inurbato, il protagonista portiano si trova innanzitutto a doversi ripetutamente difendere dalla violenza delle inquisizioni su «nome, cognome e patria» (PS XIV 29, e con minime variazioni in PS XIV 30) perpetrate in nome di una legge che sembra funzionale soltanto alla sopraffazione del povero innocente:

Chi sont?, respondi franco, in dove voo?

Sont galantomm e voo per el fatt mè;

Intuitù poeu del mestee che foo,

Ghen ven quaj cossa de vorell savè?

$[\ldots]$

Ma lu el torna de capp a interrogamm

In nomo della legge, e el solta el foss,

$E$ in nomo della legge, già se sa,

Sansessia, vala ben?, boeugna parlà. ${ }^{\text {" }}$

Inutile, per Giovannin come per Renzo, tentare di riaffermare la propria dignità di galantuomini: ${ }^{12}$ «Me par d'avegh parlaa de fioeu polid, / n'eel vera?» lamenta invano il primo («Mi pare di avergli parlato da bravo figliolo, nevvero?», Desgrazzi, v. 37), e il secondo si accoda, con la medesima vis interlocutoria, nella sua reprimenda contro l'oste della Luna piena: «Che soddisfazione, che sugo, che gusto... di mettere in carta un povero figliuolo? Parlo bene, signori? Gli osti dovrebbero tenere dalla parte de' buoni figliuoli... [...] I poveri figliuoli, n'è vero? dico bene?» (PS XIV 55-56). E a proposito dei guai di Renzo con «carta, penna e calamaio» (la terna si ripropone quasi ossessivamente in PS XIV 38, 42 e 46, ma è originariamente riferita allo scrittoio

Ma il tipo si chiaroscura nelle pieghe psicologiche di chi, raccontando le proprie disgrazie, si dà un tono» (Pietro Gibellini, Introduzione a Porta, Poesie, cit., p. XIII).

II Desgrazzi, vv. 3I-34 e 45-48 («"Chi sono?", rispondo franco, "dove vado? Sono un galantuomo e vado per il fatto mio"» "[...] ma lui torna in capo a interrogarmi "in nomo della legge", e salta il fosso, $e$ "in nomo della legge", già si sa, sia che si sia, va bene?, bisogna parlare»; «Cosa volete? è legge: anche noi bisogna ubbidire» avrebbe parimenti sentenziato l'oste in PS XV 6). La stessa scena si replica quindi nelle seconde Desgrazzi: «Ecco lì che compar el respettor / Che addirittura el comenza a interrogann, / Chi semm e chi no semm, in dove stemm, / Patria, porta, cà e tecc, e che mestee femm» («ecco lì che compare l'ispettore, che incomincia addirittura a interrogarci, chi siamo e chi non siamo, dove stiamo, patria, porta, casa e tetto, che mestiere facciamo", vv. 349-352).

I2 Come esempio delle numerose occorrenze del termine in bocca a Renzo si potrebbe citare PS XV 36: «Io? Io sono un galantuomo»; mentre sul versante portiano si segnala anche un componimento in settenari tronchi senza titolo datato al I8IO-I8II (il numero I60 nella successione di Isella): «Ch'el ghe diga pur giò ciar / Che mì sont on bon fioeu, / Galantomm, che l'è inscì rar / A trovann al dì d'incoeu» ("Gli dica pure giù chiaro che io sono un buon figliolo, galantuomo, che è così raro trovarne al giorno d’oggi», vv. 7-IO). 
di don Abbondio in PS VIII I9), varrà la pena ricordare che nel portiano On miracol (I8I3-I8I4) «Carimaa, carta, penna e do balanz» (v. 2I) sono le armi che l'Arcangelo Michele impugna accanto ad un temibile Cristo giudice, per poi riaffiorare nel contesto di un'altra "resa dei conti" in un componimento realizzato anch'esso entro il I8I4: "Alto scià penna carta e carimaa / E giustemm el nost cunt» (7O, vv. I-2). «Proppi vera, Lustrissem, che i battost / In pront come la tavola di ost» («Proprio vero, illustrissimo, che le batoste sono pronte come la tavola degli osti», Desgrazzi, vv. 65-66), verrebbe da commentare con i toni dello sfortunato Giovannin.

Non stupirà allora incontrare anche nel Bongee - pur in un contesto d'impronta decisamente più comica - le medesime reazioni impulsive e disperate del primo uomo manzoniano, che finiscono per tradursi in propositi di vendetta forse meno sanguinari ma certo non del tutto dissimili da quelli di Renzo, e in qualche modo ugualmente tacitati da un soccorso improvviso ed efficace:

\section{E se nol fudess staa che i pover mort M'han jutaa per soa grazia a tornà indree, Se no ciappi on poo d'aria, senza fall Sta voeulta foo on sparposet de cavall!!3}

Nel caso di Renzo, si sa, la «tentazione di fare uno sproposito» (PS VI 30, ma riferito al protagonista il termine ricorre già in PS II 37) è vinta dall'affacciarsi improvviso del pensiero di Lucia e «degli ultimi ricordi de' suoi parenti» (PS II 50); e l'affinità con i «pover mort» di Giovannin risulta ancor maggiore nella lezione originaria del Fermo («Si ricordò [...] gli avvisi di suo padre, le preghiere ripetute e sollecite di sua madre moribonda», I II 57-58) e, all'estremo opposto, in uno scambio di battute tra i due promessi ormai ricongiunti nel lazzaretto: «Per carità, Renzo, per carità, per i vostri poveri morti» (PS XXXVI 49). Nel vivo del confronto non si potrà nemmeno passare sotto silenzio la fugace confessione di un altro indimenticabile personaggio portiano, vale a dire il Lament del Marchionn di gamb avert (I8I6):

In quell stat de passion, de primm bullor

Me sarev fors scannaa mì come on can,

Se a tegnimm la man

No me vegneva in ment quell car amor. ${ }^{14}$

I3 Desgrazzi, vv. I35-I38 («e se non fosse stato che i poveri morti mi hanno aiutato, per grazia loro, a tornare indietro, se non prendo un po' d'aria, senza fallo sta volta fo uno sproposito bestiale»). Così anche nelle Olter desgrazzi: «Chè grazia ai pover mort no gh'è nessun / Che po' cusamm d'avegh storgiuu on cavell» («ché grazie ai poveri morti non c'è nessuno che può accusarmi d'avergli torto un capello», vv. I9-20), e ancora «S'ciavo, pascienza per i pover mort» (v. 4I7).

I4 Vv. 985-988 («In quello stato di passione, di primo impeto mi sarei forse scannato io come un cane, 
Svaniti i propositi più o meno seri di vendetta, per entrambi resta soltanto un tenace desiderio di giustizia, che cerca un'impossibile soddisfazione e, nell'attesa, si rivela nell'esigenza di raccontare i torti subiti e di guadagnare la solidarietà dei propri interlocutori, reali o ideali che siano: «Catto! In Milan, diseva intra de mì, / Gh'è giustizia, e ghe n'è tant che sia assee, / E quando me sentiran ghe giughi el coo / Che ona sattisfazion ghe l'avaroo», ${ }^{15}$ recita tra sé il Bongee, quasi approdando al celebre «a questo mondo c'è giustizia finalmente!» di PS III 60 e $62,{ }^{16}$ ma precorrendo anche le aspettative alimentate in Renzo dall'esito iniziale della rivolta dei forni («giacché oggi s'è visto chiaro che, a farsi sentire, s'ottiene quel che è giusto», PS XIV 8). L'inevitabile disillusione cova tuttavia dietro l'angolo, e si sintetizza infine nella conclusione sconsolata di Giovannin di fronte agli interessi di classe di chi dovrebbe amministrare la giustizia: «Proppi vera che can no mangia can» (Olter desgrazzi, v. 360). La battuta è talmente calzante da lasciar ipotizzare che Manzoni l'abbia raccolta e riproposta letteralmente - al di là di un eventuale ricorso poligenetico al medesimo proverbio - come un tassello di saggezza tra i commenti disparati della folla sull'intervento di Ferrer e sul destino del vicario di provvisione («il lupo non mangia la carne del lupo», PS XIV 4). ${ }^{17}$

Sempre all'ombra di un'osteria milanese, un altro personaggio portiano va quindi ad incarnare un tratto della personalità di Renzo tra i più familiari ai lettori dei Promessi sposi: in un Brindes composto nel I8Io, il popolano Meneghin mostra infatti a propria volta una vena poetica ispirata dalle generose mescite ( $« \mathrm{E}$ me senti anch mì poetta, / E voo via, via, via / Col cervell lontan di mia», con tutte le implicazioni di significato assunte anche in questo caso dalla qualifica poetta), ${ }^{18}$ per poi domandare, in un componimento omonimo di cinque anni più tardi, «Vin nostran, vin di noster campagn, /

se a trattenermi la mano non mi veniva in mente quel caro amore»: da notare che lo stesso Renzo, dopo la notte milanese, chiederà perdono «d'essere andato a dormire come un cane, e peggio", $P S$ XVII 2I).

15 «"Catto! In Milano", dicevo tra me, "c’è giustizia, e ce n'è tanto che basti; e quando mi sentiranno ci gioco la testa che una soddisfazione ce l'avrò"» (Olter desgrazzi, vv. 34I-344).

I6 In questo caso, l'accostamento è già stato proposto da Isella, Porta e Manzoni..., cit., p. 252, e nel commento di Bezzola ai Poemetti, cit., ad locum.

I7 La versione manzoniana è la precisa traduzione della locuzione milanese can no mangia can offerta da Francesco Cherubini, Vocabolario Milanese-Italiano, Milano, Imperial Regia Stamperia, I84I (consultato nell'edizione anastatica a cura di Luciano Chignoli, Milano, Libreria Meravigli Editrice, 1997), come ricorda anche il citato commento Raimondi-Bottoni, ad locum.

I8 «E mi sento anch'io poeta, e vo via, via via col cervello, lontano delle miglia» (Brindes de Meneghin a l'ostaria, vv. 30-32); un confronto con l'episodio manzoniano della Luna piena, per certi aspetti analogo, è brevemente accennato nei commenti di Raimondi-Bottoni e Nigro, ad locum, e quindi negli apparati dell'edizione del romanzo a cura di Angelo Stella e Cesare Repossi (Torino, Einaudi-Gallimard, 1995, ad locum). 
Ma legittem, ma s'cett, ma sinzer» (vv. 96-97), in anticipo sul «buon fiasco di vino sincero» chiesto dal protagonista in PS XIV 24, ma soprattutto sul «vino sincero, sano, fatto in coscienza» ordinato da Fermo in FL III VII 58. E restando nel campo della poesia - stavolta nel senso più letterale del termine - ci si potrebbe rifare persino all'esperimento di Traduzione dell'«Inferno» di Dante condotto dal giovane Porta nei primi anni dell'Ottocento e all'arduo banco di prova delle terzine dantesche, rimodellate (con la grazia che è privilegio esclusivo dei grandi) sull'universo linguistico del dialetto e sull'orizzonte di vita dei suoi umili parlanti del secolo decimonono. Se ne avrà un saggio immediato, tra i tanti possibili, nell'animato scambio fra Dante e Virgilio che chiude il Canto I: «Fermet, ghe dighi, siet mò n'ombra o on omm, / Juttem, che te fee on tratt de galantomm» (vv. 87-88) incalza il pellegrino, prima di domandare, con parole che si direbbero di Renzo, se il Paradiso promesso sia «Dove gh'è el ver paes della cuccagna?» (v. IO4), mentre la guida lo ammonisce, dal canto suo, con fare da don Abbondio ( Se mai poeù te saltass in coo di grij», v. I65). ${ }^{19}$

Da Porta a Manzoni, nel setaccio dei lettori restano tessere di lingua e frammenti d'invenzione. Ma una tessera dopo l'altra si delinea un retroterra linguistico che continua ad agire sul realismo manzoniano, e di frammento in frammento i prelievi portiani si ricuciono in racconto e vanno a testimoniare la lunga, fiduciosa ricerca «di un genere nuovo» - come ha spiegato mirabilmente Gibellini - «che narrando storie parli della vita vera, quello che troverà Manzoni col romanzo»: un genere in cui ogni singolo elemento stilistico rivela un'esigenza «di caratterizzazione etica e mimetica» e, in virtù di essa, un'inedita solidarietà (fatta «di comprensione, di una simpatia non superficiale») tra gli autori e i personaggi che prestano loro la propria voce. ${ }^{20}$ Può accadere allora che in un sonet to d'occasione, composto Per el matrimoni del sur Pepp Vandell nel I8Io, i pensieri dello stesso Porta vadano a coincidere con quelli che il narratore degli Sposi promessi immagina formulati dal suo Renzo (seppur con una qualche mediazione):

Chè se ghe fuss quaj ciall, quaj insolent,

Ch'el voress sostegnigh che el matrimoni

L'è el sepolcher d'amor, foeura di dent

Ch'el ghe diga ch'hin bozzer, ch'hin fandoni. (vv. 5-9)

I9 «Che sia il paese di cuccagna questo?» è la domanda di uno stupefatto Renzo al suo primo ingresso a Milano (PS XI 63); il rimprovero di don Abbondio percorre, invece, l'intero romanzo: «V'è saltato il grillo di maritarvi...» (PS II I7), «Se quelli che restano non metton giudizio questa volta, e scacciar tutti i grilli dalla testa, non c'è più altro che la fine del mondo» (PS XXXIII 56).

20 Si rimanda a Gibellini, Introduzione a Porta, Poesie, cit., pp. LVI, LIX-LXIII. 
E se, dopo vent'anni, Renzo avesse trovato in uno di questi bei libri, che il talamo è tomba d'amore; si sarebbe fatto interpretar la sentenza, perchè alle parole non ci arrivava; ma intesala bene, avrebbe sentenziato dal canto suo che l'autore di quella dovess'essere un sempliciotto che non conoscesse le cose di questo mondo. (SP III XXXVIII 64) ${ }^{21}$

Il gioco di rifrazioni tra i microcosmi paralleli di Porta e Manzoni si moltiplica, d'altro canto, nelle pagine illustrate delle edizioni gemelle della Quarantana e delle Poesie scelte portiane: date coincidenti (I840-1842), medesimo editore (Guglielmini e Redaelli), stessa équipe di disegnatori e incisori, non si faticherà a scorgervi in filigrana un progetto unitario, in cui si specchia nitidamente il consorzio umano comune alle opere dell'uno e dell'altro. ${ }^{22}$ Lo si dovrebbe forse prendere come un suggerimento implicito a soffermarsi rispettosamente su quelle immagini, sugli scorci affollati di popolo o sull'intimità dei ritratti della Tetton e di Lucia, ${ }^{23}$ piuttosto che cercare ad ogni costo di tradurre la sensazione di familiarità in deduzioni logiche e dipendenze immediate. Un ultimo, interessante banco di prova potrebbe essere, in proposito, il poemetto La guerra di pret, lasciato incompiuto da Porta nel I820, a un passo dalla sua morte e ad un soffio dall'avvio del romanzo di Manzoni. $\mathrm{Ci}$ sarebbe più di un elemento in supporto della tentazione di rileggerne la trama in chiave manzoniana, a partire dal nucleo propulsivo di una povera montanara insidiata e rapita, in questo caso invero da un abate, che «aveva pettaa i oeucc su la Luvisa, / Ch'el ghe girava e barzegava intorna / E el ghe tendeva i ret per falla amisa». ${ }^{24}$ Ma sarà meglio, anche stavolta, attenersi a prelievi più minuti e puntuali: ascoltare Porta che descrive il persecutore «incazzii [...] de la passion, / Del comod de vedella, de inzigalla, / Desperaa daj refud, daj repetton, / Da no podè nè toeulla nè impattalla», ${ }^{25}$ lasciando che si riattivi la memoria di don Abbondio che mette in guardia da don Rodrigo perché «È un signore quello, con cui non si può né vincerla né impattarla» (PS XXV 5I); ${ }^{26}$ o contemplare la sfortunata Luvisa prigioniera in una

2I «Ché, se ci fosse qualche sciocco, qualche insolente che volesse sostenere che il matrimonio è il sepolcro dell'amore, gli dica fuori dai denti che sono storie, sono fandonie».

22 Si confronti, al riguardo, Isella, Carlo Porta..., cit., pp. 247-248.

23 La caratteristica dominante dei «cavij a la zoeura, / Spartii in duu su la front, negher e folt» («i capelli alla montanina spartiti in due sulla fronte, neri e folti», Lament del Marchionn di gamb avert, vv. 229-230) e le altre affinità tra le due figure sono già state indicate, in particolare, nel commento di Bezzola ai Poemetti, cit., p. 395.

24 Vv. I28-130 («aveva messo gli occhi sulla Luisa, [...] le girava e bazzicava d'intorno e le tendeva le reti per farsela amica»).

25 Vv. I45-I48 («incarognito [...] dalla passione, dall'agio di vederla, di tentarla, disperato dai rifiuti, dalle ripulse, dal non poter né vincerla né impattarla»).

26 I commenti di Raimondi-Bottoni e Poggi Salani (Alessandro Manzoni, I Promessi sposi. Testo del I840-I842, a cura di Teresa Poggi Salani, Milano, Centro Nazionale di Studi Manzoniani, 2013, ad locum) rimandano soltanto all'equivalente espressione milanese, di uso comune. 
torre, «pù morta che nè viva, / Senza speranza de soccors uman» (vv. 206207), un po' come Lucia avrebbe raccontato nel Fermo di essersi trovata nel castellaccio «lontana da ogni soccorso... senza speranza di liberazione» (IV VIII 25). E riconoscere il medesimo, raro privilegio di un'intima conoscenza e di una spontanea capacità di restituzione dell'animo umano persino in un singolo verso: «Ah che pur tropp el coeur l'è ona gran spia» (v. 229). «Certo il cuore ne dice tante» avrebbe abbozzato di lì a poco Manzoni nel getto iniziale dell'ispirazione ${ }^{27}$ prima di formulare quel celebre passaggio da cui, da allora, spira per tutti aria di casa: «Certo, il cuore, chi gli dà retta, ha sempre qualcosa sa dire. Ma che sa egli il cuore? Appena un poco di quello che è già accaduto» (FL I VIII 48, poi, quasi invariato, in PS VIII 88).

federica.alziati@unifr.ch / federica.alziati@unicatt.it

27 Si consultino, in proposito, gli apparati dell'edizione critica di riferimento del Fermo e Lucia, ad locum. 